\title{
Are Preoperative Systemic Immune Index and Neutrophil-to-Lymphocyte Ratio Sufficient to Predict Lymph Node Positivity and Overall Survival in Muscle- Invasive Bladder Cancer Cases?
}

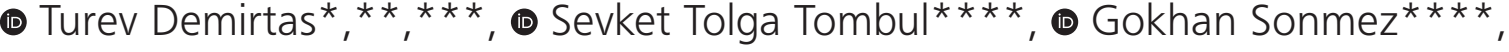 \\ (1) Abdullah Demirtas $* * * *$ \\ *Erciyes University Medical Faculty Department of Medical History and Ethics, Kayseri, Turkey \\ **Erciyes University Faculty of Medicine, Department of Stem Cell Sciences, Genome and Stem Cell Center, Kayseri, Turkey \\ ***Erciyes University, Halil Bayraktar School of Occupational Health, Emergency Medicine Program, Kayseri, Turkey \\ $* * * *$ Erciyes University Faculty of Medicine, Department of Urology, Kayseri, Turkey
}

\section{Abstract}

\begin{abstract}
Aim: The neutrophil-lymphocyte ratio (NLR) is a parameter that has been shown to be effective as a prognostic marker in many solid tumors. It is aimed to investigate NLR and the systemic immune-inflammation index (SII) in predicting the overall survival and lymph node positivity in bladder cancer (BC).

Methods: The retrospective study included patients that underwent radical cystoprostatectomy/radical cystectomy (RC) due to muscleinvasive bladder cancer (MIBC), high-grade T1 BC, or carcinoma in situ in our clinic between January 2010 and March 2020. All the patients had no history of preoperative metastasis, lymph nodes, chemotherapy, hematological malignancies, and preoperative urinary tract infection. Data on neutrophil, lymphocyte, platelet, and hemoglobin levels and total white blood cell counts were retrieved from clinical records and data on disease stage and lymph node positivity were retrieved from pathology reports. Follow-up times and survival times were recorded.

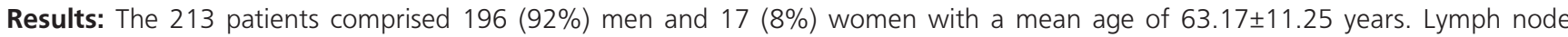
positivity was detected in 49 (23\%) patients. The mean overall survival time was 75.04, 63.77, and 84.4 months in all patients, patients with lymph node positivity, and patients with lymph node negativity, respectively. No significant difference was found between patients with lymph node positivity and negativity with regard to NLR and SII values ( $p=0.975$ and $p=0.745$, respectively). In the receiver operating characteristics (ROC) analysis NLR and SII had no significant effect in predicting lymph node positivity [Area under the ROC curve (AUC) 0.499 (95\% confidence interval (Cl): 0.403-0.594) and AUC 0.485 (95\% Cl: 0.394-0.575), respectively] and in predicting overall survival [AUC 0.423 (95\% Cl: 0.346-0.501, $p=0.056)$ and AUC 0.435 (95\% Cl: 0.357-0.514, $p=0.107)$, respectively]
\end{abstract}

Conclusion: The results indicated that SII and NLR are not sufficient to predict lymph node positivity and survival in patients with organ-confined BC

Keywords: Bladder cancer, neutrophils, lymphocytes, lymph nodes, systemic immune-inflammation index

\section{Introduction}

Bladder cancer (BC) is among the most frequently seen cancers in the world, ranking the $7^{\text {th }}$ most common cancer in men and $11^{\text {th }}$ in women (1). In Turkey, BC is the $4^{\text {th }}$ most common cancer in men and $13^{\text {th }}$ in women (2). At the time of diagnosis, $70-75 \%$ of the cases are non-muscle invasive bladder cancer (NMIBC). In the first five years after diagnosis, 50-70\% of NMIBC cases will recur. On the other hand, $10-15 \%$ of NMIBC cases progress to muscle-invasive bladder cancer (MIBC) and approximately $25 \%$ of BC cases are MIBC at the time of diagnosis. The five-year survival rate in MIBC is only $50 \%(3,4)$. Radical cystectomy $(R C)$ is the most effective treatment in MIBC. Regardless, some patients may recur. Therefore, neoadjuvant chemotherapy

Address for Correspondence: Abdullah Demirtas, Erciyes University Faculty of Medicine, Department of Urology, Kayseri, Turkey Phone: +90 5325094494 E-mail: mesane@gmail.com ORCID: orcid.org/0000-0001-9102-5518 Received: 06.05.2021 Accepted: 09.06.2021 
is recommended particularly in patients with T2-T4a, NOMO tumors in order to increase overall survival and diseasefree survival. Nevertheless, this therapy has been shown to provide positive contributions only to overall survival (5). To our knowledge, there are no reliable indicators showing patient suitability for neoadjuvant chemotherapy and/or patient prognosis after cystectomy.

Accumulating evidence suggests that immune response cells (monocytes, lymphocytes, and neutrophils) and platelets along with their associated signaling pathways are an important component of the tumor microenvironment and that these factors can play a significant role in tumor progression and metastasis (6). In particular, the effectiveness of neutrophil-to-lymphocyte ratio (NLR), platelet-to-lymphocyte ratio (PLR), and C-reactive proteinto-albumin (CRP/ALB) ratio in predicting recurrence and life expectancy has been investigated (7-9). These studies have shown that the systemic immune-inflammation index (SII), which is calculated by multiplying the NLR ratio by the number of platelets, has a prognostic value in solid tumors such as gastric and colorectal esophageal tumors and also in BC. The role of NLR and SII in predicting the prognosis of prognosis BC is already known (10-14). Also, higher SII was shown to be related to prostate cancer with a high Gleason score (15). Moreover, lymph node positivity is known as an indicator of poor prognosis in BC (3).

In this study, we aimed to determine whether NLR and SII values are effective in predicting the overall survival and lymph node positivity in BC.

\section{Methods}

This retrospective study was approved by Erciyes University Medical School Clinical Research Ethics Committee (date: June 10, 2020; no: 220/273). Patients with complete follow-up data and who underwent radical cystoprostatectomy/RC due to MIBC, high-grade T1 BC, or carcinoma in situ in our clinic, between January 2010 and March 2020 were evaluated retrospectively. Patients whose age was between 18-85 years old, who had no solid organ and lymph node metastasis on preoperative imaging [computed tomography (CT), magnetic resonance imaging (MRI), or positron emission CT (PET-CT)], without history of bladder sparing protocol and without the indication of salvage cystectomy due to conditions such as hematuria and postrenal acute renal failure, without a known history of hematological malignancies, neoadjuvant chemotherapy within the last one month and preoperative diagnosis of cystitis/pyelonephritis were included. Having the known history of hematological malignancies and neoadjuvant chemotherapy, having indication for salvage cystectomy due to persistent symptoms of BC despite chemotherapy performed according to the bladdersparing protocol, being below 18 years old and above 85 years old, having preoperative diagnosis of cystitis and/or pyelonephritis were settled as exclusion criteria.

In all patients included in the study, data on neutrophil, lymphocyte, platelet, and hemoglobin levels and total white blood cell counts were retrieved from clinical records and data on disease stage and lymph node positivity were retrieved from pathology reports. NLR and SII values were calculated as previously described in the literature (14). Follow-up durations and overall survival times were calculated from follow-up data. Overall survival was defined as the time from surgery to death. The 2017 TNM classification was used for tumor staging (16). Informed consent was obtained from each patient.

\section{Statistical Analysis}

Data were analyzed using SPSS for Windows version 22 (Armonk, NY: IBM Corp.). The normal distribution of continuous variables was assessed using the KolmogorovSmirnov test. Continuous variables with normal distribution were expressed as mean \pm standard deviation median and continuous variables with nonnormal distribution were expressed as median $\left(25^{\text {th }}-75^{\text {th }}\right.$ percentile). Categorical variables were expressed as frequencies (\%). Continuous variables with nonnormal distribution were compared using the Mann-Whitney $U$ test. The prognostic values of NLR and SII in predicting lymph node positivity were calculated using the area under the receiver operating characteristics (ROC) curve (AUC).

\section{Results}

Of the 475 patients evaluated, 213 patients that met the inclusion criteria were included in the study. These patients comprised 196 (92\%) men and 17 (8\%) women with a mean age of $63.17 \pm 11.25$ years. Lymph node positivity was detected in $49(23 \%)$ patients, while no surgical margin positivity was present in any patient (Table 1).

No significant difference was found between patients with lymph node positivity and negativity with regard to NLR and SII values ( $p=0.975$ and $p=0.745$, respectively). In the ROC analysis that was performed to evaluate the effectiveness of NLR and SII in predicting lymph node positivity, the AUC value for NLR and SII was 0.499 [95\% confidence interval $(\mathrm{Cl}): 0.403-0.594$ and $0.48595 \% \mathrm{Cl}$ : 0.394-0.575], respectively. These values indicated that NLR and SII had no effect in predicting lymph node positivity (Table 2, Figure 1).

The mean overall survival time was 75.04, 63.77, and 84.4 months in all patients, patients with lymph node positivity, and patients with lymph node negativity, respectively. No significant difference was found between patients with lymph node positivity and negativity with regard to overall survival $(p=0.227)$. In contrast, the 


\begin{tabular}{|l|l|}
\hline \multicolumn{2}{|l|}{ Table 1. Characteristics of patients } \\
\hline Variables & $\mathbf{( n = 2 1 3 )}$ \\
\hline Age (years) & $63.17 \pm 11.25$ \\
\hline Sex & $17(8 \%)$ \\
\hline Male & $196(82 \%)$ \\
Female & \\
\hline Pathological stage $\mathbf{( n ,} \%)$ & $12(5.6 \%)$ \\
\hline T0 & $5(2.3 \%)$ \\
Tis & $7(7.3 \%)$ \\
Ta & $22(10.3 \%)$ \\
T1 & $8(3.8 \%)$ \\
T2a & $68(31.9 \%)$ \\
T2b & $38(17.8 \%)$ \\
T3a & $36(36 \%)$ \\
T3b & $14(6.6 \%)$ \\
T4a & $2(0.9 \%)$ \\
T4b &
\end{tabular}

Type of the urinary diversion applied

\begin{tabular}{|l|l|}
\hline $\begin{array}{l}\text { Cutaneous incontinent urinary } \\
\text { diversion } \\
\text { Orthotopic bladder }\end{array}$ & $146(69 \%)$ \\
\hline Follow up in month & $67(31 \%)$ \\
\hline $\mathbf{5}$ year survival $(\mathbf{n}, \%)$ & $74.9(12.7-78.4)^{*}$ \\
\hline Overall survival with $\mathbf{9 5} \% \mathbf{C l}$ & $75.04(63.22-86.85)$ \\
\hline${ }^{*}$ Median $\left(25^{\text {th }}-7^{\text {th }}\right.$ percentile) Cl: Confidence interval \\
\hline
\end{tabular}

Table 2. Comparison of SII, NLR and survivals according to lymph node status

\begin{tabular}{|l|l|l|l|}
\hline & $\begin{array}{l}\text { Lymph node positive } \\
(\mathbf{n = 4 9 )}\end{array}$ & $\begin{array}{l}\text { Lymph node } \\
\text { negative } \\
(\mathbf{n = 1 6 4 )}\end{array}$ & $\mathbf{p}$ \\
\hline NLR & $2.57(1.87-3.69)^{*}$ & $2.67(2.04-3.52)$ & $0.975^{* *}$ \\
\hline SII & $700(474.9-876.5)$ & $678.5(502.1-1025.1)$ & $0.745^{* *}$ \\
\hline $\begin{array}{l}5 \text { year } \\
\text { survival } \\
\text { rate }\end{array}$ & $10 / 49(20.4 \%)$ & $64 / 164(39.0 \%)$ & $0.016^{* * *}$ \\
\hline $\begin{array}{l}\text { Overall } \\
\text { survival }\end{array}$ & $63.77(29.32-98.21)$ & $84.40(72.13-96.66)$ & $0.227^{* * *}$ \\
\hline $\begin{array}{l}* \text { Median (25th-75 } \\
\text { Systemic immune-inflammation index, NLR: Neutrophil-to-lymphocyte ratio, }\end{array}$ \\
\hline
\end{tabular}

mean five-year survival rate was $34.7 \%, 20.4 \%$, and $39 \%$, respectively, and a significant difference was found between patients with lymph node positivity and negativity $(p=0.016)$. In the ROC analysis performed to evaluate the effectiveness of NLR and SII in predicting survival, the AUC value was 0.423 (95\% Cl: 0.346-0.501, $p=0.056)$ and 0.435 (95\% Cl: 0.357-0.514, $p=0.107)$, respectively. Accordingly, NLR and SII were found to have no significant effect in predicting survival (Figure 2,3).

\section{Discussion}

The present study investigated the effect of NLR and SII in predicting lymph node positivity, which is inflammatory indicators calculated from preoperative blood parameters, in patients who underwent RC due to clinical T1/T2 NOM0

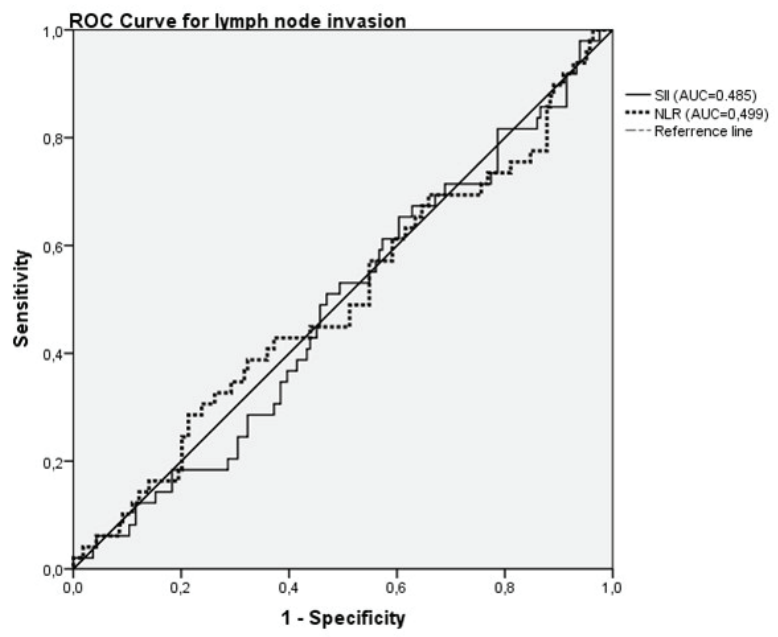

Figure 1. ROC curve for lymph node invasion ROC: Receiver operating characteristics, SII: Systemic immuneinflammation index, NLR: Neutrophil-to-lymphocyte ratio

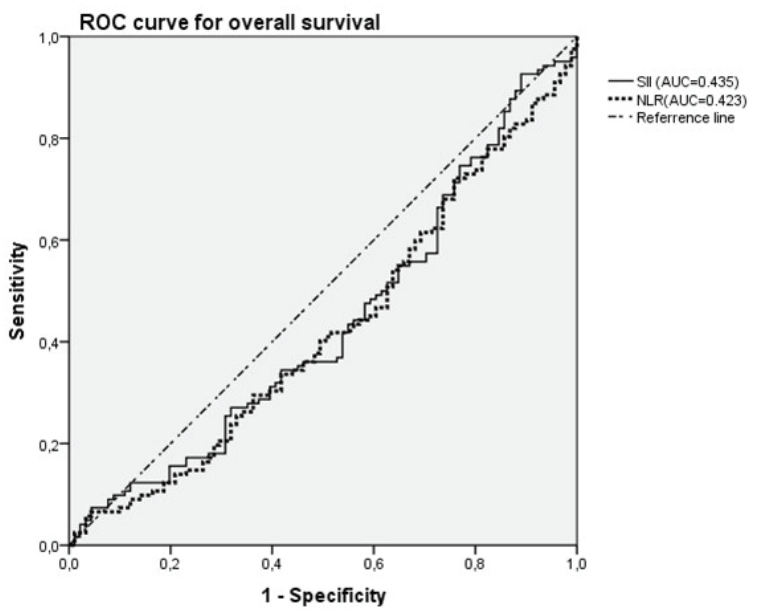

Figure 2. ROC curve for overall survival

ROC: Receiver operating characteristics, SII: Systemic immuneinflammation index, NLR: Neutrophil-to-lymphocyte ratio

MIBC. The median follow-up period was 29.9 months. Although no significant difference was found between patients with lymph node positivity and negativity with regard to overall survival time (63.77 and 84.4 months, respectively) $(p=0.227)$, the five-year survival rate was higher in patients with lymph node negativity $(p<0.05)$. No significant difference was found between the two groups with regard to NLR and SII values ( $p=0.975$ and $p=0.745$, respectively). Moreover, ROC analysis indicated that NLR and SII had no effect in predicting lymph node positivity and overall survival. To our knowledge, our study is the first of its kind to show that SII and NLR have no significant effect in predicting lymph node positivity in patients with MIBC. 


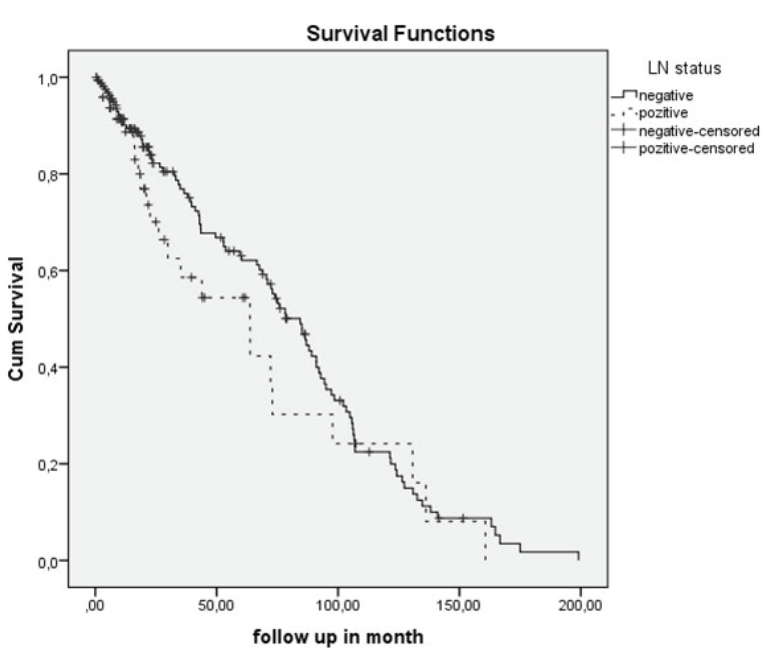

Figure 3. Overall survival according to lymph node (LN) statusKaplan-Meier curve

Gorgel et al. (13) evaluated a cohort of 191 patients who underwent RC and reported that SII values greater than 843 were cancer-specific and indicated a poor overall survival. However, the authors did not state whether they only included organ-confined MIBC. Additionally, the authors did not perform an analysis regarding the prediction of lymph node positivity (13). Similarly, Zhang et al. (14) evaluated a patient series of 209 patients that were randomized into primary $(n=139)$ and validation $(n=70)$ cohorts and reported that SII is an independent factor in predicting overall survival and is more effective than NLR. However, the researchers examined the effect of SII and NLR only in predicting overall survival. Similarly, the authors did not state whether they only included organ-confined MIBC (14). Bi et al. (17) found that peripheral nutritional index (PNI) and SII are independent factors in predicting overall survival in high-risk PT1 patients. Nevertheless, the group analyzed in that study consisted of 387 patients who received intracavitary Bacillus Calmette-Guérin (BCG) treatment due to high-risk PT1, and only 42 of the patients underwent RC. Accordingly, this patient group had a better prognosis when compared to the patients evaluated in the present study (17). In a retrospective study, Peng et al. (18) evaluated 516 patients who underwent RC and reported that PNI and NLR were independent factors in predicting overall survival and progression-free survival. In that study as well, the authors did not state whether they only included patients with organ-confined MIBC, and they performed no evaluation regarding the role of the investigated indicators in predicting lymph node positivity (18).

\section{Study Limitations}

The main limitations of this study were its retrospective manner and including highly selected small group of patients. The present study only included patients with organ confined MIBC and aimed to seek an answer to the following question: "If SII and NLR are effective parameters in predicting lymph node positivity, can they be used to determine candidate patients for neoadjuvant chemotherapy?". Nonetheless, the results indicated that SII and NLR had no significant effect in predicting lymph node positivity and overall survival in this patient group. We consider that this finding could be attributed to the inclusion of a highly selected patient group in our study. However, to our knowledge, it is the first study in the literature to investigate the role of SII and NLR in predicting lymph node positivity. Also with respect to the prognosis of $\mathrm{BC}$, the data about cancer specific survival could not be given in this study due to incomplete data entry about reason of death.

\section{Conclusion}

From the results obtained from this study it can be concluded that SII and NLR are not sufficient to predict lymph node positivity and survival in patients with organconfined BC. However, these results should be confirmed in prospective studies with larger cohort.

\section{Authorship Contributions}

Concept: S.T.T., A.D., Design: S.T.T., A.D., Data Collection or Processing: S.T.T., G.S., Analysis or Interpretation: S.T.T., G.S., T.D., Literature Search: S.T.T., G.S., T.D., Writing: S.T.T., A.D.

Conflict of Interest: No conflict of interest was declared by the authors.

Financial Disclosure: The authors declared that this study received no financial support.

\section{References}

1. Bray F, Ferlay J, Soerjomataram I, Siegel RL, Torre LA, Jemal A. Global cancer statistics 2018: GLOBOCAN estimates of incidence and mortality worldwide for 36 cancers in 185 countries. CA Cancer J Clin 2018;68:394-424.

2. Kara F, Keskinkılınç B. Türkiye Kanser İstatistikleri 2016, Halk Sağlığı Genel Müdürlüğü, Sağlık Bakanlığı. Ankara,2019. (www.hsgm.saglik.gov.tr/depo/birimler/kanser-db/istatistik/ Turkiye_Kanser_statistikleri_2016.pdf) Accessed at 20 July 2020

3. Cumberbatch MGK, Jubber I, Black PC, et al. Epidemiology of bladder cancer: a systematic review and contemporary update of risk factors in 2018. Eur Urol 2018;74:784-95.

4. Marcos-Gragera R, Mallone S, et al. Urinary tract cancer survival in Europe 1999-2007: Results of the populationbased study EUROCARE-5. Eur J Cancer 2015;51:2217-30. 
5. Witjes JA, Bruins M, Cathomas R, et al. EAU Guidelines on Muscle-Invasive and Metastatic Bladder Cancer. Edn. presented at the EAU Annual Congress Milan 2021. p 40-56. ISBN 978-94-92671-07-3. Publisher: EAU Guidelines Office. Place published: Arnhem, The Netherlands.

6. Hanahan D, Weinberg RA. Hallmarks of cancer: the next generation. Cell 2011;144:646-74.

7. Marchioni M, Primiceri G, Ingrosso M, et al. The Clinical Use of the Neutrophil to Lymphocyte Ratio (NLR) in Urothelial Cancer: A Systematic Review. Clin Genitourin Cancer 2016;14:473-84.

8. Kaynar M, Yıldırım ME, Badem $\mathrm{H}$, et al. Bladder cancer invasion predictability based on preoperative neutrophillymphocyte ratio. Tumour Biol 2014;35:6601-5.

9. Guo Y, Cai K, Mao S, et al. Preoperative C-reactive protein/ albumin ratio is a significant predictor of survival in bladder cancer patients after radical cystectomy: a retrospective study. Cancer Manag Res 2018;10:4789-804.

10. Shi $H$, Jiang $Y, C a o ~ H$, et al. Nomogram Based on Systemic Immune-Inflammation Index to Predict Overall Survival in Gastric Cancer Patients. Dis Markers 2018;2018:1787424.

11. Xie QK, Chen $P$, et al. The systemic immune-inflammation index is an independent predictor of survival for metastatic colorectal cancer and its association with the lymphocytic response to the tumor. J Transl Med 2018;16:273.

12. Geng Y, Shao Y, Zhu D, et al. Systemic Immune-Inflammation Index Predicts Prognosis of Patients with Esophageal
Squamous Cell Carcinoma: A Propensity Score-matched Analysis. Sci Rep 2016;6:39482.

13. Gorgel SN, Akin Y, Koc EM, Kose O, Ozcan S, Yilmaz Y. Retrospective study of systemic immune-inflammation index in muscle invasive bladder cancer: initial results of single centre. Int Urol Nephrol 2020;52:469-73.

14. Zhang W, Wang R, Ma W, et al. Systemic immune-inflammation index predicts prognosis of bladder cancer patients after radical cystectomy. Ann Transl Med 2019;7:431.

15. Sonmez G, Demirtas T, Tombul ST, Akgun H, Demirtas A. Diagnostic efficiency of systemic immune-inflammation index in fusion prostate biopsy. Actas Urol Esp (Engl Ed) 2021;45:359-65.

16. TNM classification of malignant tumors. UICC International Union Against Cancer. 8th edn., G.M. Brierley JD, Wittekind C, Editor. 2017, Wiley-Blackwell and UICC: New York, USA. Available from: https://www.uicc.org/sites/main/ files/private/TNM_Classification_of_Malignant_Tumours_ Website_15\%20MAy2011.pdf

17. Bi H, Shang Z, Jia C, et al. Predictive Values of Preoperative Prognostic Nutritional Index and Systemic ImmuneInflammation Index for Long-Term Survival in High-Risk NonMuscle-Invasive Bladder Cancer Patients: A Single-Centre Retrospective Study. Cancer Manag Res 2020;12:9471-83.

18. Peng D, Gong YQ, Hao $H$, et al. Preoperative Prognostic Nutritional Index is a Significant Predictor of Survival with Bladder Cancer after Radical Cystectomy: a retrospective study. BMC Cancer 2017;17:391. 